\title{
Lamellar orientation and interlamellar cracks in co-crystallized poly(ethylene oxide)/poly(L-lactic acid) blend
}

\author{
Ya-Ting Hsieh, Siti Nurkhamidah and Eamor M Woo
}

The correlation between the lamellar orientation in ring-banded spherulites and crack patterns in blends of two crystalline polymers crystallized in two steps was investigated. The two polymers were poly(ethylene oxide) (PEO) and poly(L-lactic acid) (PLLA) with a low molecular weight. The techniques used for this investigation included polarizing light optical microscopy, scanning electron microscopy and atomic force microscopy. Crack formation was influenced by the ring band patterns in PLLA and overlapping or impingement between PEO spherulites upon cooling. By extracting the water-soluble PEO from the PEO/PLLA blend, the inner morphology of the lamellar textures was further revealed to have a complex pattern. It consisted of the outer macro-lamellae with a flat-on width of $20 \mu \mathrm{m}$ and twisting micro-lamellae with a 1-2 $\mu \mathrm{m}$ edge-on width. As the excess PEO was etched off from the valley and the interlamellar crevices of the PLLA lamellae, the outer macro-lamellae plates ( $\sim 20 \mu \mathrm{m}$ flat-on width) exhibited up and down periodicity with flat-on orientation. However, the micro-lamellae (with 1-2 $\mu \mathrm{m}$ edge-on width) with twisting were visible inside the cracks. Similar analysis of a PEO-rich composition, PEO/PLLA (80/20) blend with irregular ring bands and no cracks, revealed that the outer macro-lamellae were not present. However, all micro-lamellae twists in the ridge regions irregularly protrude out to create zig-zag banding.

Polymer Journal (2011) 43, 762-769; doi:10.1038/pj.2011.63; published online 27 July 2011

Keywords: cracks; lamellae orientation; PEO; PLLA; spherulites

\section{INTRODUCTION}

Poly(L-lactic acid) (PLLA) is a biodegradable polyester with potential applications in environmental conservation. Nevertheless, it has some mechanical and processing disadvantages that have to be overcome before it could be used for these applications. Modification by blending with other polymers offers such a route. Poly(ethylene oxide) (PEO), a water-soluble polyether, is biocompatible and miscible to some polyesters $^{1,2}$ with very low toxicity in biomedical applications. ${ }^{3}$ PEO has been proven to be miscible with some amorphous polymers, for example, in classically known miscible blends of $\mathrm{PEO} /$ poly(methyl methacrylate) (PMMA) ${ }^{4}$ and PEO/poly(vinyl phenol) (PVPh). ${ }^{5}$ The phase behavior in PEO blends with semicrystalline polyesters has also been extensively studied. The phase behavior of blends of PEO/PLLA has been studied by Nakafuku et al. ${ }^{6}$ and Nijenhuis et al. ${ }^{7}$ It is also known to be miscible with semicrystalline polyesters, such as poly(3hydroxybutyric acid) (PHB), ${ }^{8}$ poly(ethylene succinate) (PESu) ${ }^{9}$ and poly(butylene succinate) (PBSu). ${ }^{10}$ Partial miscibility with phase domains in blends of PEO with PLLA of high molecular weight has been claimed. ${ }^{6,7}$ Reportedly, blending PEO with PLLA can further enhance the biocompatibility of PLLA; ${ }^{11,12}$ however, separate domains in both the amorphous and crystalline domains in PEO/PLLA might make it complicated to addressing the biocompatibility.
Cracks in some semicrystalline polymers have been reported during their cooling or crystallization processes or when they were in contact with solvents. Two types of cracks have been reported in PHB: radial cracks and circumferential (concentric) cracks. ${ }^{13}$ To interpret these cracks, the coefficients of thermal expansion in two different directions (radial versus transverse) of spherulites have been used. Martinez-Salazar et al. ${ }^{14}$ attempted to attribute the cause of circumferential cracks to the coefficients of thermal expansion in the radial direction was greater than that in the circumferential direction, which led to the formation of concentric circumferential cracks. Preliminarily studies have been conducted on the crack behavior in neat PHB was. ${ }^{15,16}$ In yet another case, poly(trimethylene terephthalate) (PTT) has been reported to exhibit circumferential cracks when it was in contact with solvents. This observation may have some correlation with the ringbanded pattern in the PTT spherulite. ${ }^{17}$ Solvent effects or thermal shrinkages due to different coefficients of thermal expansions might be responsible for the cracks. However, the full picture on crack behaviors has yet to be constructed for complete interpretation because not all ring-banded polymer-spherulites will show cracks.

An earlier study indicated that molecular weight, crystallinity and crystalline morphology developed at the crystallization temperature $\left(T_{\mathrm{c}}\right)$ in PLLA were all factors that could influence the crack pattern on 
cooling. Both PEO and PLLA are crystalline polymers and spherulite growth of these two polymers may impinge and overlap on each other, leading to complex interferences and behaviors between cracks and ring bands in the spherulites grown in the blends. Using a model blend of PEO with PLLA, this study first attempted to prove the phase behavior in the amorphous phase. The phase behavior of the PEO/ PLLA blend has been briefly discussed in the literature, ${ }^{18}$ and most studies have claimed that the PEO/PLLA blend might be partially miscible depending on the molecular weight of the PLLA. A preliminary study in this laboratory proved that a homogeneous liquid phase could be achieved between PEO and PLLA if the PLLA molecular weight was kept low. Furthermore, PEO in blends could be easily dissolved in water. To enhance contrast, PEO could be wateretched from PEO/PLLA blends, leaving the PLLA lamellae unaltered. More recently, crack mechanisms and crack patterns in neat PLLA with a low molecular weight were explored in greater details, ${ }^{19}$ which showed significantly more types of cracks than the circumferential type of crack reported earlier. For PHB crystallizing on PLLA as templates, the lamellar orientation of ring-banded spherulites, $T_{\mathrm{c}}$ and crystallinity have all been proven as major factors influencing the types of microcracks on the surface of co-crystallized crystalline/crystalline blends. ${ }^{20}$

The objective of this study was to probe the mechanisms of cracks and ring bands in PLLA spherulites co-crystallizing with PEO crystal phases. In the crystalline/crystalline PEO/PLLA blend, PLLA spherulites are ring-banded within a certain $T_{c}$ range, whereas $\mathrm{PEO}$ spherulites are non-banded at any $T_{\mathrm{c}}$. It would be novel and useful to explore how cracks in the PEO/PLLA blend might be influenced by the co-crystallizing growth of the ring-banded PLLA and non-banded PEO spherulites. The crack behavior in PLLA is a critical issue, but it has not been sufficiently studied. PEO is miscible with low-molecularweight PLLA, and both PEO and PLLA are capable of developing crystallinity. However, the co-crystallized structure of PEO can be easily etched off. Therefore, this study aimed at identifying the detailed mechanisms of cracks in two crystalline phases of PEO and PLLA and at elucidating the elusive mechanisms governing the formation of cracks. It also aimed to clarify the possible correlation between the behavior of cracks and ring bands in semicrystalline polymers.

\section{EXPERIMENTAL PROCEDURE}

\section{Materials and preparation}

PEO $\left(M_{\mathrm{w}}=20000 \mathrm{~g} \mathrm{~mol}^{-1}, T_{\mathrm{g}}=-60^{\circ} \mathrm{C}\right.$ and $\left.T_{\mathrm{m}}=64^{\circ} \mathrm{C}\right)$ was obtained from Aldrich (St Louis, MO, USA). PLLA $\left(M_{\mathrm{w}}=11000 \mathrm{~g} \mathrm{~mol}^{-1}, T_{\mathrm{g}}=45^{\circ} \mathrm{C}\right.$ and $T_{\mathrm{m}}=155^{\circ} \mathrm{C}$ ) were purchased from Polyscience, Inc. (Warrington, PA, USA). Sample films of PLLA and PEO blends were prepared by solution-casting using chloroform as the common solvent. Two polymers (PLLA and PEO) with specific compositions were dissolved in the solvent with a concentration of 4 $\mathrm{wt} \%$, well stirred, and cast onto micro glass slides at $45^{\circ} \mathrm{C}$. The cast films were first dried at $45^{\circ} \mathrm{C}$ for $24 \mathrm{~h}$ to evaporate the solvent and then further dried in vacuum at $\sim 40^{\circ} \mathrm{C}$ for several days.

For polarizing light optical microscopy (POM) characterizations, the $\mathrm{PEO} /$ PLLA blend samples were either pressed into thin films between two micro glass slides or covered with a polyimide (PI) film for easy release after crystallization. All blend samples in this study were covered on the top during crystallization. Procedures involved that a small quantity of blend samples (dissolved in the solvent) was uniformly spread onto a micro glass slide and properly dried before being covered with another micro glass slide. These PEO/ PLLA samples, pressed between two slides, were then heated and pressed into thin films of proper thickness ( $7-14 \mu \mathrm{m}$ or thinner, $\sim 5 \mu \mathrm{m}$ ) on a heating stage at a maximum melting temperature $\left(T_{\max }\right)$ of $190^{\circ} \mathrm{C}$ for $2 \mathrm{~min}$ to erase previous crystals. The samples were then rapidly transferred to another heating stage pre-set at a designated $T_{\mathrm{c}}\left(105-135^{\circ} \mathrm{C}\right)$ to crystallize the films at various lengths of time. Thermogravimetric analysis was used to analyze the degradation of neat PLLA under static air, which began at $\sim 200^{\circ} \mathrm{C}$. The $T_{\max }$ was set at $190^{\circ} \mathrm{C}$ in this study because of thermal stability concerns.

Additional sets of PEO/PLLA blend samples were prepared for water etching before morphological characterizations. For this purpose, PI films were used for covering the blends during heating and crystallization. After crystallization, the PI film was peeled off, and pure water was used as the etching solvent of the PEO in the blends.

\section{Apparatus and procedures}

Polarizing optical microscopy (POM). A POM (Nikon Optiphot-2, Tokyo, Japan), equipped with a digital camera charge-coupled device and a microscopic hot stage (Linkam THMS-600, Surrey, UK) with TP-92 temperature programmer, was used for characterizing crystalline morphology of the polymers.

Scanning electron microscopy (SEM). Samples of solution-cast films of PLLA or its blends were examined using a SEM (FEI Quanta-400F, Hillsboro, OR, USA) to reveal the lamellar pattern of spherulites. Film samples intended for SEM characterization were prepared following special procedures as following. The top surfaces of the samples could not be covered by glass and had to be exposed for SEM characterization; that is, the top cover of the film samples should be easily removed after crystallization. Therefore, a PI film, instead of a micro glass slide, was used as the top cover for these film samples. After thermal treatments, the PI film on top of the sample film, which was deposited firmly on the bottom glass slide, was carefully peeled off. After the PI film was peeled off, the top surfaces of the exposed film samples on the bottom micro glass slides were then coated with gold by vacuum sputtering prior to SEM characterization.

Atomic force microscopy (AFM). AFM characterization was made in intermittent tapping mode (Caliber, Veeco-DI Corp., Santa Barbara, CA, USA) with a silicon tip $(v=70 \mathrm{kHz}, r=10 \mathrm{~nm})$. The largest scan range was $150 \mu \mathrm{m} \times 150 \mu \mathrm{m}$, and selective focusing was performed on narrower areas of $5 \mu \mathrm{m} \times 5 \mu \mathrm{m}$. Thin films were deposited on glass substrates with an open face for AFM characterization. The top surfaces of the film samples were exposed in the same way as that used in preparing the SEM samples. Preliminary optical microscopy $(\mathrm{OM})$ characterization was performed to confirm the presence of cracks or ring-band crystals in the samples intended for subsequent AFM characterization.

Fourier-transform infrared spectroscopy (FTIR). FTIR (Nicolet Magna-560, Madison, WI, USA) spectra were obtained at $4 \mathrm{~cm}^{-1}$ resolution and obtained from 64 scans (for enhanced signals) in the wavenumber range of 500$4000 \mathrm{~cm}^{-1}$. Blend samples (unetched crystallized blends and the water-etched portion) for infrared measurements were cast as thin films with uniform thickness directly on $\mathrm{KBr}$ pellets at ambient temperature. Infrared measurements were performed on samples cast or dried on $\mathrm{KBr}$ pellets.

\section{RESULTS AND DISCUSSION}

\section{Cracks and ring bands in PEO/PLLA blends}

For neat PLLA with a low molecular weight, it was already known that alternating ring bands were present in spherulites only when it was crystallized with a top cover at $T_{\mathrm{c}}=125-130^{\circ} \mathrm{C}$; the spherulites became ring less when the PLLA was crystallized at $T_{\mathrm{c}}$ lower than $125^{\circ} \mathrm{C} .{ }^{19}$ However, as PEO was blended into PLLA, ring-banded spherulites were more easily seen in all blend compositions crystallized at all $T_{\mathrm{c}} \mathrm{s}$, indicating that PEO could be present as a diluting agent and induce changes on the lamellar assembly of PLLA during crystallization. The crack patterns in the PEO/PLLA blends were found to be distinctly different from those in the neat PLLA, indicating that the crystalline PEO component in the blend influenced the lamellar assembly and the cracks. Figure 1 shows POM crystals versus OM graphs for cracks in PEO/PLLA blends $\left(T_{\mathrm{c}}=110^{\circ} \mathrm{C} 30 \mathrm{~min}\right.$ for full crystallization of PLLA and then cooled to ambient temperature) of different compositions: 

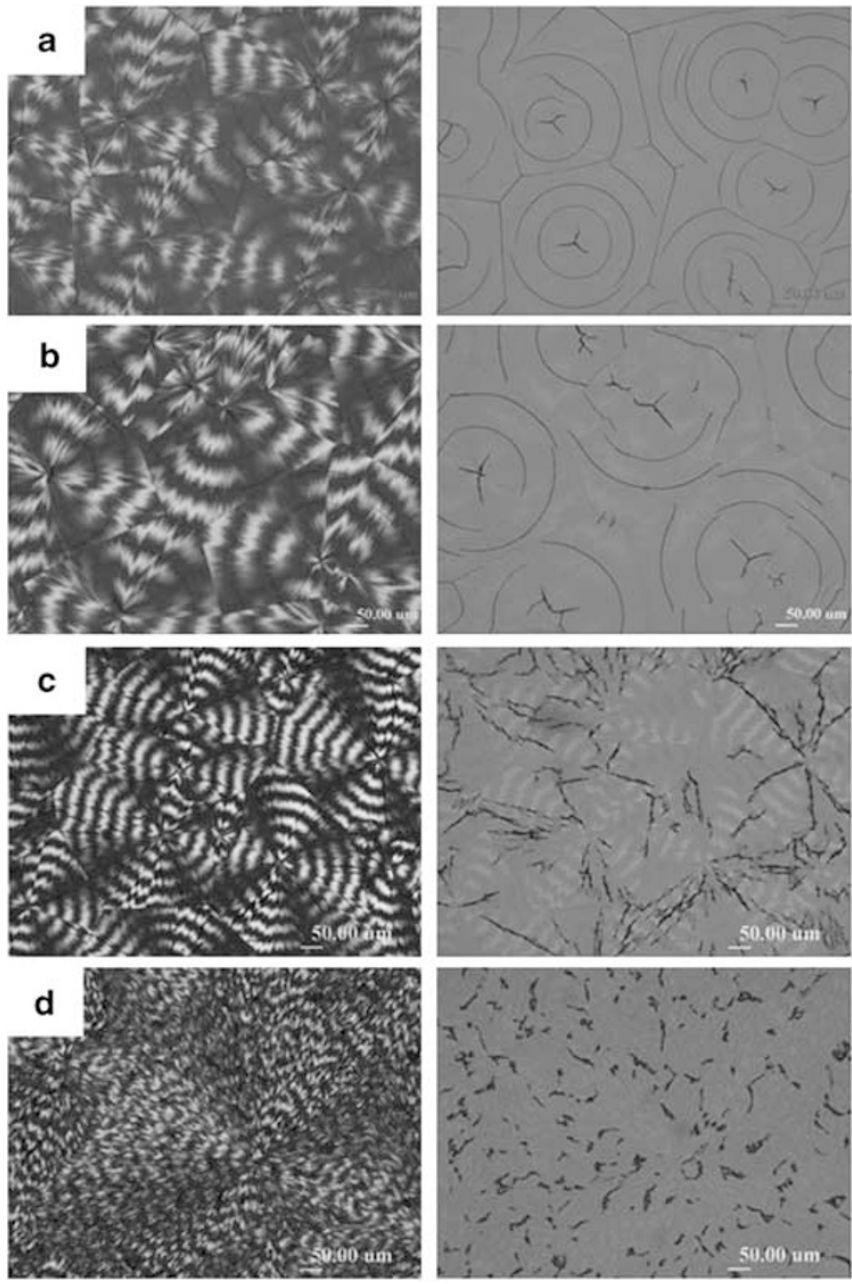

Figure 1 POM (left) versus OM (right) graphs revealing different ring bands and crack patterns in the PEO/PLLA blends crystallized at $110^{\circ} \mathrm{C}$ of compositions (a) 10/90, (b) 20/80, (c) 50/50 and (d) $80 / 20$ (the graphs were taken at ambient temperature after cooling from $T_{\mathrm{c}}$ ). A full color version of this figure is available at Polymer Journal online.

(a) 10/90, (b) 20/80, (c) 50/50 and (d) 80/20. Similar to neat PLLA, cracks in the blends did not appear at the $T_{\mathrm{c}}$ of PLLA crystallization. However, cracks appeared when the PEO/PLLA blends were cooled to ambient temperature $\left(25-30^{\circ} \mathrm{C}\right)$, at which the PEO component in the blends also crystallized. All POM or OM graphs in this figure were taken at ambient temperature after cooling from $T_{\mathrm{c}}$. Crack patterns in spherulites, grown at $T_{\mathrm{c}}$ then cooled to ambient temperature, apparently changed with respect to PEO fractions in the PEO/PLLA blends. For the PLLA-rich blends ( $\mathrm{PEO}=10$ and $20 \mathrm{wt} \%$ in graphs $\mathrm{a}$ and $\mathrm{b}$ ), only continuous and concentric circumferential-type cracks were present, and the cracks mainly coincided with the dark bands of the rings. In addition, tiny tri-branched cracks were seen at the nuclei where the spherulite growth originated. However, for the PEO-rich blends $(\mathrm{PEO}=50$ and $80 \mathrm{wt} \%$ in graphs $\mathrm{c}$ and $\mathrm{d})$, concentric circumferential-type cracks were no longer present; instead, only radial or short random types of cracks were seen in the spherulites. In the POM graph for the PEO/PLLA (50/50) blend shown in Figure $1 \mathrm{c}$, the ring bands were still regular but the spherulites were highly impinged. Correspondingly, the cracks in the spherulites of the

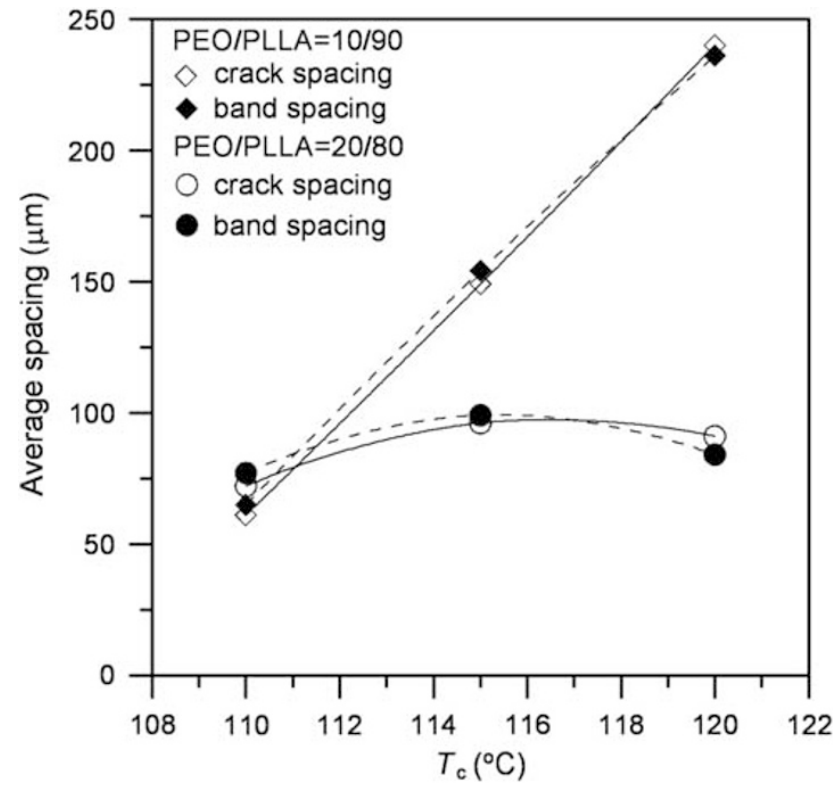

Figure 2 Correlation plot between the ring-banded spacing and the intercrack width in the PEO/PLLA blends (10/90 and 20/80) crystallized at $T_{\mathrm{c}}=110-120^{\circ} \mathrm{C}$ (the film thickness was kept at $7-14 \mu \mathrm{m}$ ).

50/50 blend were mainly along the radial direction. For comparison, the POM graphs for the PEO/PLLA (80/20) blend in Figure 1d showed highly distorted ring bands and randomly oriented cracks. The crack types in the spherulites of the blends seemed to be intricately associated with the ring band patterns in the same spherulites, and the lamellae patterns responsible for the ring bands were likely the main causes of the observed cracks.

In the entire PEO/PLLA blend compositions (10/90, 20/80, 50/50 and 80/20) crystallized at the specified $T_{\mathcal{c}}$, ring bands were always present in the spherulites. However, crack patterns differed significantly and varied with respect to compositions. Note that for neat PLLA, ring bands appeared in spherulites when it was crystallized at $T_{c}=125-130{ }^{\circ} \mathrm{C}$, as reported in the literature ${ }^{19}$ However, the range of $T_{\mathrm{c}}$ for ring bands in the miscible PLLA and PEO blends, which had a lower $T_{\mathrm{g}}$, was shifted and slightly widened; the lower bound of $T_{\mathrm{c}}$ was from 110 to $130^{\circ} \mathrm{C}$. In general, the figure shows that the regularity and inter-ring spacing decreased as the PEO content increased in the PEO/ PLLA blends. The crack types in the blends at ambient temperature, as discussed above, were apparently influenced by the ring-band patterns formed at $T_{\mathrm{c}}$. The relationship between the cracks and ring bands in crystalline polymers and blends needs to be better understood.

To further clarify such phenomenon, interspacing of the ring bands and corresponding circumferential cracks were measured and correlated. Figure 2 shows a correlation plot between the ring-banded spacing and the inter-crack spacing in the PEO/PLLA blends crystallized at $T_{\mathrm{C}}=110-120^{\circ} \mathrm{C}(t=30 \mathrm{~min}$, and the film thickness was at $7-14 \mu \mathrm{m})$. The data show that the inter-spacing of the cracks and ringbands agreed well with each other, indicating that the dark bands of the ring bands generally coincided with the circumferential cracks. The evidence indicates that the circumferential cracks tended to occur at the interfaces of alternating bright-dark bands, and, thus, the periodicity of the cracks coincided with the periodicity of the ring bands.

In general, the rule of matching periodicity mentioned above holds true at $T_{\mathrm{c}}=110-115^{\circ} \mathrm{C}$, where ring bands are clear and circular. At higher $T_{\mathrm{c}}\left(T_{\mathrm{c}}=\sim 115-120^{\circ} \mathrm{C}\right)$, where ring bands are still regular and 
circular, the inter-spacing of ring-bands and the inter-spacing of cracks widen significantly in a synchronizing way. The exception to this rule of matching ring-band and crack inter-spacing was observed only when the sample films were exceptionally thin (below $\sim 5 \mu \mathrm{m}$ ), as exemplified in additional laboratory work not shown here for brevity. This observation indicates that the formation of the ring bands in polymer films requires a minimum thickness. The effect of film thickness on ring-banded morphology is another issue; but when film thickness is kept at $7-14 \mu \mathrm{m}$, the correlation between ring bands and cracks is quite clear. It should be briefly noted here that the thickness of the sample film affects the ring patterns owing to growth diffusion hindrance. The ring patterns in turn affect the crack patterns in the polymers or blends.

\section{Impinged spherulites of PEO/PLLA blends}

In addition to the above correlation between cracks and ring bands in the blends, other observations were also noted. For blends with PEO contents $<20 \mathrm{wt} \%$, cracks appeared when the blends were cooled to ambient temperature. This behavior was similar to the neat PLLA samples, and the crack patterns were mainly smooth and concentric. Furthermore, the cracks precisely coincided with the dark band of the alternating bright-dark ring bands of the spherulites. However, the crack behavior was different in the blends with high PEO contents ( $>50 \mathrm{wt} \%$ ). The PEO had an interfering effect as miscible amorphous chains or discrete crystal phases on the PLLA cracks in the blend spherulites. For the PEO/PLLA blends with PEO contents $>50 \mathrm{wt} \%$ or more, cracks did not form immediately upon cooling to ambient temperature because the PEO in the miscible blends crystallized slowly at these temperature. Instead, irregular or radial cracks appeared as the PEO in the blends gradually crystallized. Although the ring bands in PLLA spherulites appeared regular and clear, the cracks in the blends were significantly disrupted. The fact suggests that as both PLLA and PEO crystallize sequentially, the cracks in the PEO/PLLA blends were influenced not only by the ring-banded crystals of PLLA but also by impingement of the PLLA spherulites with the crystals of PEO, leading to a highly irregular crack pattern in the blends with high $\mathrm{PEO}$ contents. These irregular cracks were apparently influenced by multiple factors, such as the earlier-formed PLLA lamellae, the subsequent PEO crystals, the impinging growth fronts of both crystals and the entrapment of amorphous PEO chains in the PLLA lamellae owing to the blend miscibility. More detailed discussions and analysis are in the following sections.

As discussed earlier, the PEO-rich PEO/PLLA blends (50/50 and $80 / 20$ ) exhibited radial or random types of cracks and not circumferential ones. It was interesting to probe the mechanisms that led to such different cracks in the PEO-rich PEO/PLLA blends. POM characterization was performed on the $80 / 20$ blend, which was subjected to a two-step crystallizations process. For the first step, the process lasted for $7 \mathrm{~min}$ to partially crystallize the PLLA at $T_{\mathrm{c}}=110^{\circ} \mathrm{C}$. For the second step, the blend was cooled to ambient temperature for various time periods (from several seconds up to $1 \mathrm{~min}$ ). Figures $3 \mathrm{a}-\mathrm{d}$ show the POM graphs of the PEO/PLLA (80/20) blend illustrating the evolution of the ring bands of the PLLA spherulites in the first step (at $T_{\mathrm{c}}$ ) and those of the PEO spherulites in the second step (at ambient temperature). The blend was kept at ambient temperature for (a) $9 \mathrm{~s}$, (b) $12 \mathrm{~s}$ and (c) $21 \mathrm{~s}$. Cracks were present along with the ring bands in the spherulites of the blend, but the cracks could not be clearly observed in the POM graphs. The OM graph for sample $\mathrm{c}$ is shown in Figure $3 \mathrm{~d}$ to reveal its crack patterns. Similar procedures were taken to reveal cracks in sample a and b. For brevity, not all samples are shown. When held at $T_{\mathrm{c}}=110^{\circ} \mathrm{C}$, only the PLLA phase in blends crystallized
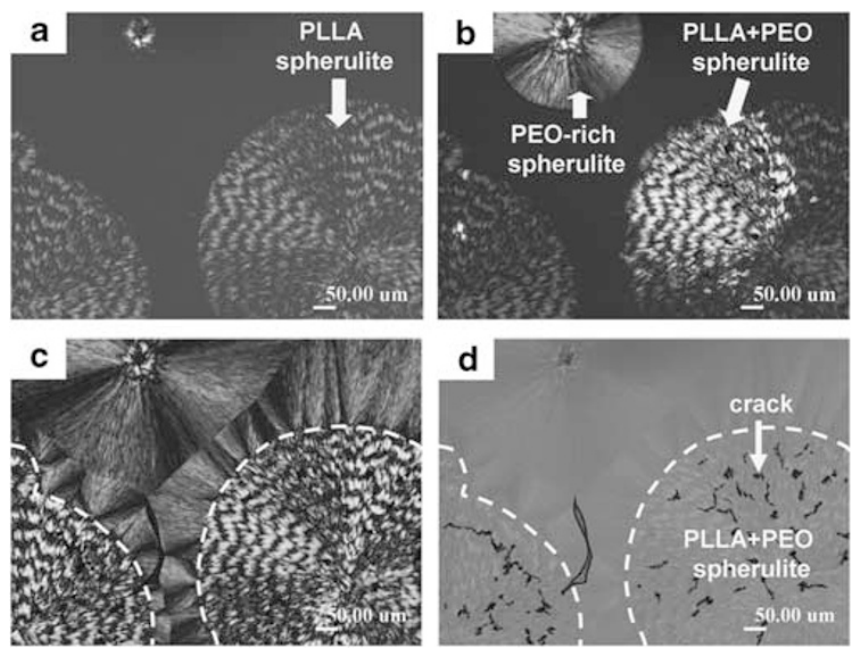

Figure $3 \mathrm{POM}$ graphs revealing the two types of spherulite and the cracks in the PEO/PLLA (80/20) blend. The blend was crystallized at $T_{\mathrm{c}}=110^{\circ} \mathrm{C}$ and then cooled to ambient temperature and kept for (a) $9 \mathrm{~s}$, (b) $12 \mathrm{~s}$ and (c) $21 \mathrm{~s}$. (d) OM graph of sample c revealing cracks in the overlapping PEO/ PLLA spherulites. A full color version of this figure is available at Polymer Journal online.

into spherulites. Ring bands could be clearly observed, but no cracks developed. When the blend was crystallized at $T_{\mathrm{c}}=110^{\circ} \mathrm{C}$, the PLLA component showed characteristic zig-zag ring-bands with large interring spacings in the spherulites. However, a portion of the PEO crystallized inside the PLLA spherulites along the crystal templates of the PLLA lamellae when it was cooled from $T_{\mathrm{c}}$ to the ambient temperature and held for periods of time. In the meantime, portions of PEO gradually developed separate PEO spherulites that eventually overlapped and impinged with the PLLA spherulites formed at an earlier $T_{\mathrm{c}}$. The progressive growth of PEO along with the impinging or overlapping PEO crystals are shown in Figures $3 \mathrm{a}-\mathrm{c}$. The brightness of the PLLA spherulites intensified on the overlapped growth of PEO inside, which indicates that the PEO lamellae growing along the original PLLA lamellae intensifies the birefringence rather than nullifying it. To view the cracks in the spherulites of the blends more clearly, non-polarizing OM graphs were taken in addition to the POM graphs. Figure 3d shows the OM graph for sample c, revealing cracks only in regions where the PEO spherulites grown later overlapped the PLLA spherulites grown earlier. No cracks were observed in either neat PEO spherulites or neat PLLA spherulites without any overlap. When the crystallization time was extended at the ambient temperature, the PEO spherulites continued to grow in size and number, and their boundary were seen to extend into the PLLA crystal regimes. Cracks appeared in regimes where two different crystals were in contact with or impinging or overlapping each other. In the regions where PLLA crystallized instead of PEO, no cracks were observed; thus, the presence of cracks in blends with high PEO contents was determined by PEO crystallization and PEO spherulites superimposing on the PLLA crystals grown earlier. These cracks were typically irregular owing to the randomly overlapped domains and they seemed to mark the interfaces.

However, POM or OM results were not sufficient to reveal finer details of the lamellae in the dark or bright bands. In the following section, the morphology of the etched samples is further discussed using SEM and AFM results at higher magnifications. 


\section{Morphology of PEO-etched blends}

Figure 4 shows the SEM photos of spherulites in water-etched PEO/ PLLA (80/20) blend after it was isothermally crystallized at $110^{\circ} \mathrm{C}$ : (a) the lamellae with disordered rings at a lower magnification, and (b) the seaweed-like PLLA lamellae at a higher magnification. As most of the PEO component was etched out, the remaining lamellae in the etched spherulite should be mostly PLLA. In this PEO-rich PEO/PLLA blend (80/20), PLLA chains were highly diluted. On crystallization, the PLLA lamellae were thin (1-2 $\mu \mathrm{m}$ in width) with mostly edge-on orientation. These thin PLLA lamellae tended to interpenetrate with the PEO crystals during growth before the PEO was etched. After the PEO was etched, most of the exposed PLLA lamellae in the PEO-rich blend $(\mathrm{PEO} / \mathrm{PLLA}=80 / 20)$ seemed to have an edge-on orientation with occasional twisting.

Figure 5 shows the SEM images for the spherulites of the $\mathrm{PEO} /$ PLLA (50/50) blend isothermally crystallized at $110^{\circ} \mathrm{C}$ : (a) sample
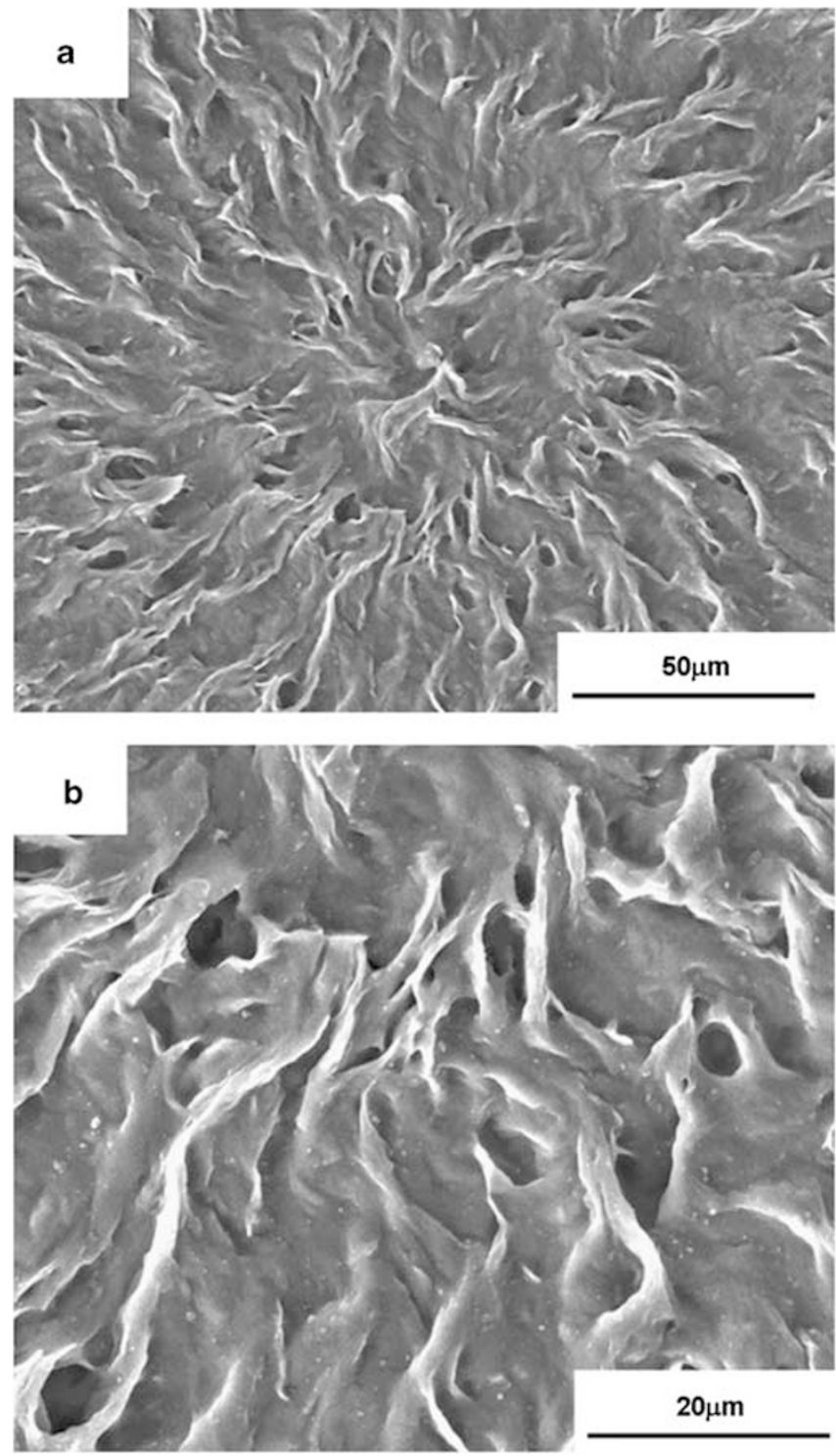

Figure 4 SEM graphs of spherulites in water-etched PEO/PLLA (80/20) after it was isothermally crystallized at $110^{\circ} \mathrm{C}$ : (a) the lamellae with disordered rings at a lower magnification, (b) the seaweed-like poly(L-lactic acid) lamellae at a higher magnification. before water etching, and (b) sample after water etching. The morphology images were all taken at ambient temperature. For the unwashed PEO/PLLA blend samples, the SEM graph in Figure 5a shows no height changes between the dark and bright bands. After the samples were washing, Figure $5 \mathrm{~b}$ shows that the bright ring bands were crowded with many radial direction cracks/voids, where each of the short cracks assumed a lenticular shape $\sim 23 \mu \mathrm{m}$ long (approximately equal to the band width of the bright band) and a crack width of up to $5 \mu \mathrm{m}$. The samples were etched to preferentially remove the PEO from the blend and to help reveal the inner morphology. Other than bowing up from valley to ridge to form a periodic convex shape, the top layer of lamella on the bright band apparently did not turn or twist. However, sub-lamellar crystals with various twists and orientations were observed inside the cracks. In addition, after washing, the height profile became significant. The SEM graphs of the etched blend (50/50) clearly showed that some lamellae inside the cracks were
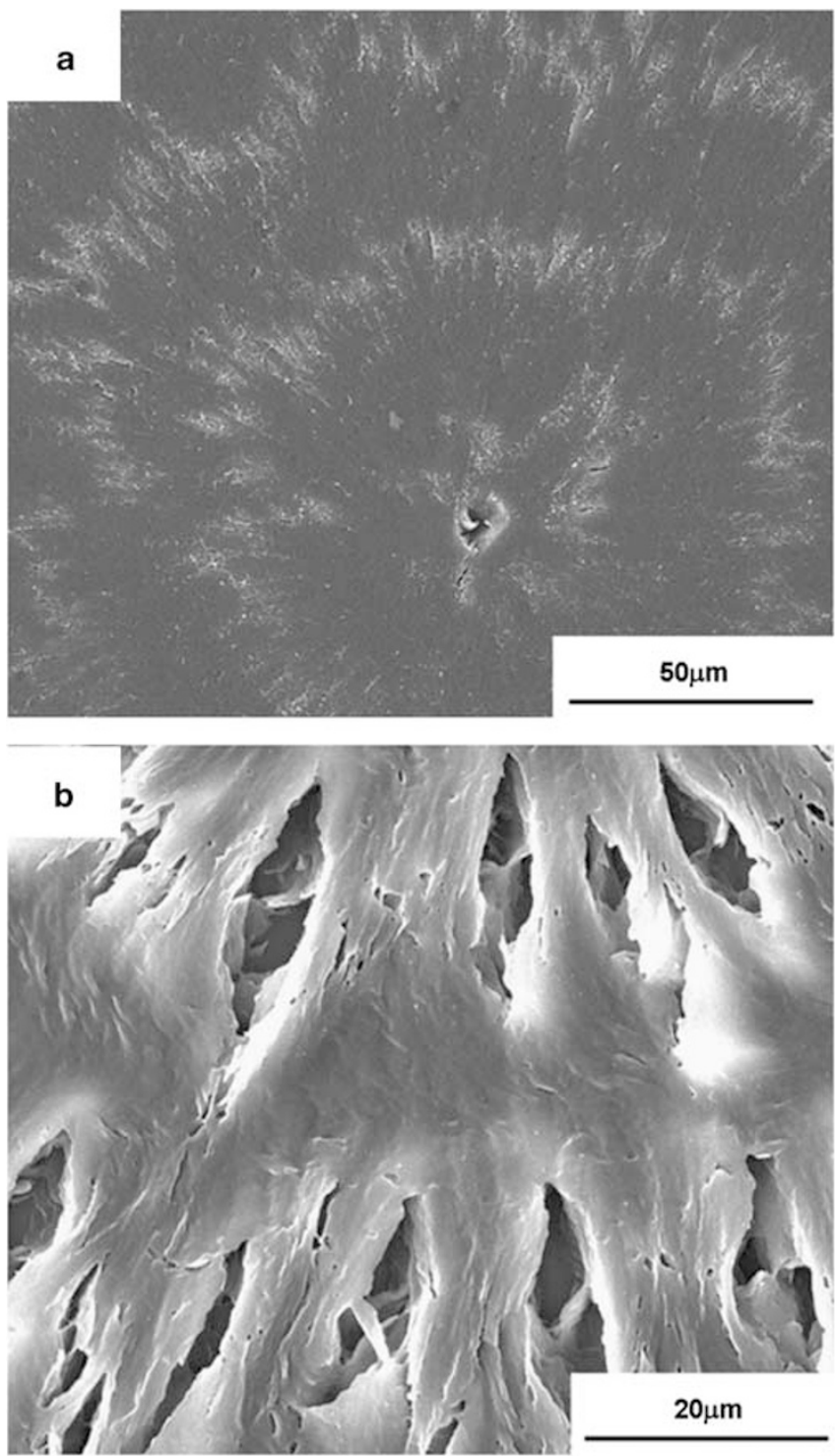

Figure 5 SEM graphs for the spherulites in the PEO/PLLA (50/50) blend isothermally crystallized at $110^{\circ} \mathrm{C}$ : (a) as crystallized/unwashed and (b) water-etched to expose the poly(L-lactic acid) lamellae. 
twisted between the edge-on and flat-on arrangements. Interestingly, the external top shells on the bright bands, which were separated by the lenticular shape cracks, were still distinctly flat-on lamellar plates and similar to the lamellae in the valley of the dark bands. The bright bands were composed of short radial cracks, twisted and untwisted lamellae, and flat-on lamellae that were all confined in the bright bands. AFM height profile characterization, to be discussed in greater details later, revealed that the dark bands were indeed circumferentially etched almost to the substrate surface.

The results above clearly show that the ring-banded pattern in the PEO/PLLA blends of 80/20 and 50/50 (PEO-rich versus equal composition, respectively) persisted even after washing. Before the samples were etched, the blend surfaces were flat. After etching, the textures reflecting the ring-banded patterns were longitudinal, crater-like valleys. Furthermore, the OM graphs for the unetched sample revealed that the bright ring bands, where radial short-segmental cracks were clearly exposed and visible, were not seen prior to washing. After etching, the ring bands became crater-like. This observation indicates that the dark bands must be composed of species that could be more easily removed by water.

AFM was performed on the blend samples before and after water etching to characterize the quantitative height profiles and to obtain images of the blends. Before washing, the bright and dark bands in the blends had the same height. Figures 6 shows the AFM results for the water-etched PEO/PLLA (50/50) blend crystallized at $T_{\mathrm{c}}=110^{\circ} \mathrm{C}$ : (a) height image at a size of $150 \mu \mathrm{m} \times 150 \mu \mathrm{m}$, and (b) height profile along the corresponding line marked on graph a. The morphological graphs were all taken at the ambient temperature. In Figure 6a numerous cracks in the radial direction were observed in the bright bands, but no cracks were observed in the dark band. In the waterwashed samples, the bright ring band was crowded with short radial cracks with an average height greater than the dark band, where no cracks were observed. Figure $6 \mathrm{~b}$ shows the height profile for washed samples along the radial direction. The bright/dark bands in the ring bands exhibited alternating up and down, with an average period of about $35 \mu \mathrm{m}$ and a height change of about 3-4 $\mu \mathrm{m}$. Apparently, water etching removed materials from the dark bands of the alternating bright/dark bands of the spherulites. Note that the height profile change was more noticeable in the washed PEO/PLLA samples. In the unwashed samples, the height change was significantly less noticeable across the periodic bright/dark bands. Similarly, the AFM height profile was measured on the unwashed PEO/PLLA (50/50) sample to compare with the water-etched PEO/PLLA sample. Figure $6 \mathrm{c}$ shows that the unwashed sample (PEO/PLLA $=50 / 50$ blend, also crystallized at $T_{\mathrm{c}}=110^{\circ} \mathrm{C}$ ), had a vertical height difference of only $\sim 0 \mu \mathrm{m}$ from valley to ridge, indicating that water etching removed an amorphous layer of at least $3 \mu \mathrm{m}$ thick from the spherulites.

After the sample was washed, the dark band became much lower than the bright one, which indicates that the entire dark band was uniformly and circumferentially etched out but that the bright band was only selectively etched along the radial cracks. Additional AFM phase image and SEM characterizations indicate that bright bands before washing were originally littered with small crevices. On washing portions of the inner structures of the bright bands were etched out, and the cracks were widened. However, the outer shells of the bright bands remained unchanged after washing. Therefore, the height of the bright bands under OM viewing remained the same, and the entire bright band appeared relatively unchanged. Nevertheless, AFM and SEM characterization revealed etching on the inner portion of the bright bands. By etching out the PEO component from the PEO/PLLA blend, the lamellar structures of PLLA were more fully exposed. Thus,

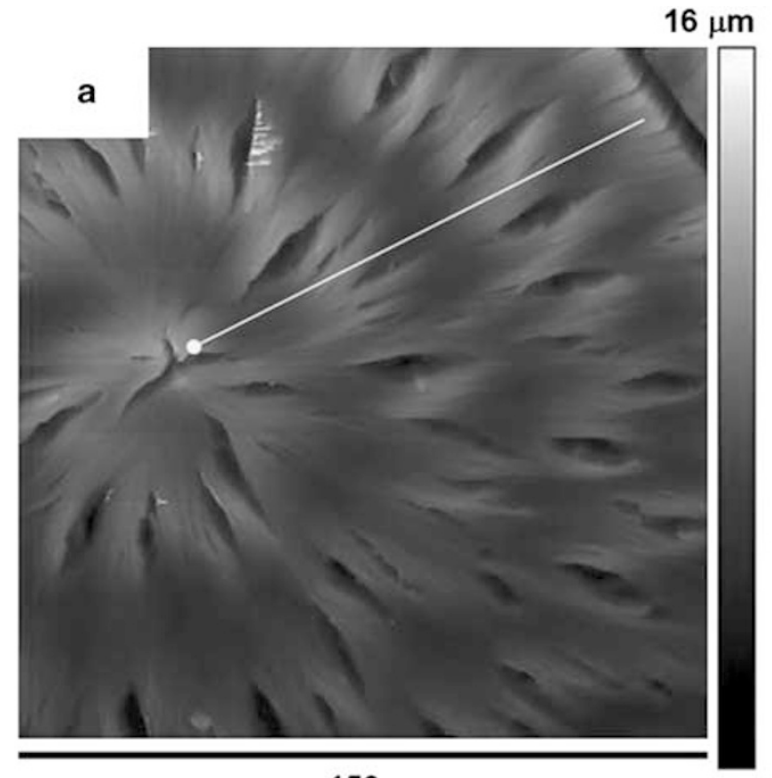

$150 \mu \mathrm{m}$
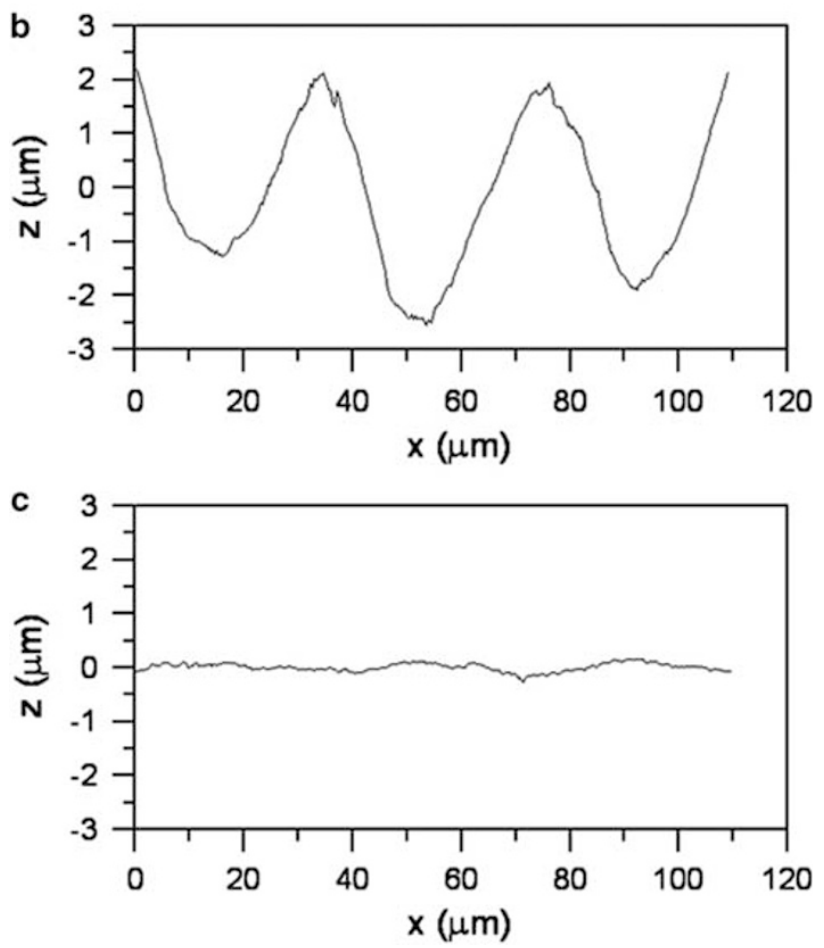

Figure 6 AFM analyses of the water-etched PEO/PLLA (50/50) blend crystallized at $110^{\circ} \mathrm{C}$ : (a) height image, $150 \mu \mathrm{m} \times 150 \mu \mathrm{m}$, (b) vertical height profile $\sim 3 \mu \mathrm{m}$ from valley to ridge and (c) the vertical height profile showing no variation from valley to ridge for the unwashed sample. A full color version of this figure is available at Polymer Journal online.

it was quite likely that the main lamellar plates on the top, radiating out from the center, grew up and down in a wavy manner as they grew outwards. The width of the plates increased as they grew and branched out from minor crystals. These minor crystals, in turn, stemmed from the main stalks occurring in certain growth spots. Periodic branching occurred underneath the convex bulk with twisting and some unavoidable cracks. Such twists and sub-cracks were observed inside the 


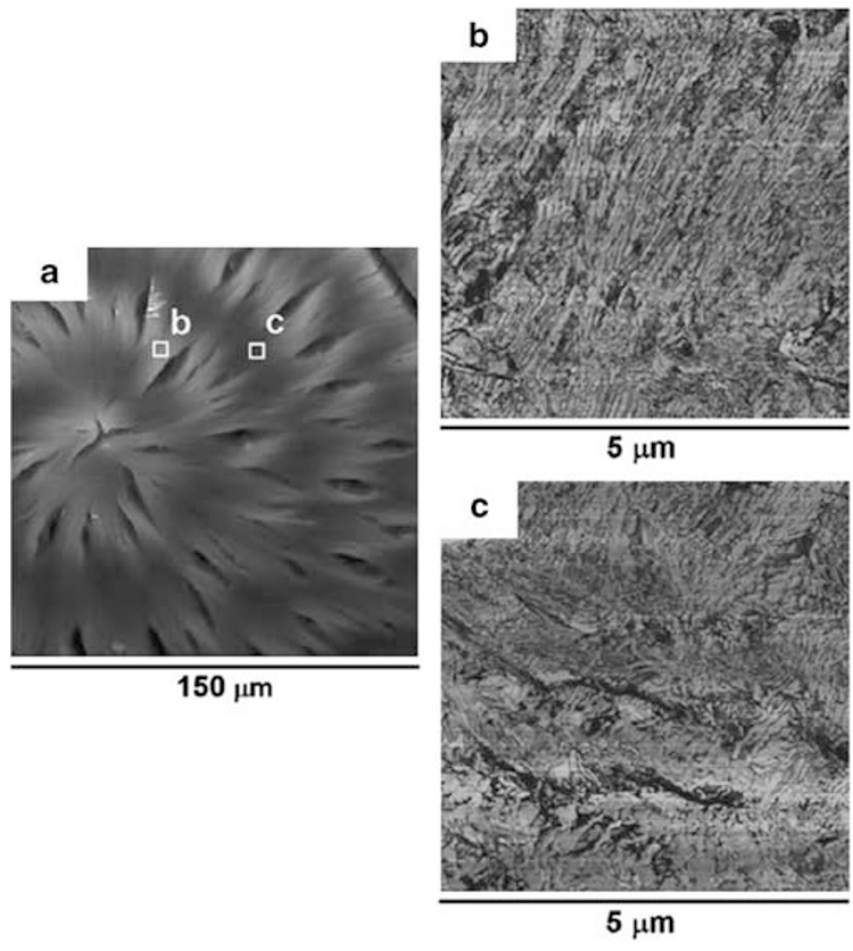

Figure 7 (a) AFM images $(150 \mu \mathrm{m} \times 150 \mu \mathrm{m})$ of water-etched PEO/PLLA (50/50) blend crystallized at $110^{\circ} \mathrm{C}$, (b) zoom-in of the ridge marked as 'region b', (c) zoom-in of the valley marked as 'region c'. A full color version of this figure is available at Polymer Journal online.

large lenticular-shaped crack openings in the water-etched PEO/PLLA blends. In the PEO/PLLA blend (50/50), the PLLA lamellae in the water-etched samples were mainly flat-on plates that were $\sim 5-15 \mu \mathrm{m}$ wide, as measured from near center to the rim. These lamellae were dramatically different from the thin edge-on lamellae, and no wide flat-on platelet lamellae were seen in the PEO-rich PEO/PLLA (80/20) blend discussed earlier.

The above AFM and SEM analyses on the etched PEO/PLLA shed new lights on the lamellar assembly packed by two crystallizing polymers in a blended state. As revealed in the SEM graphs of the water-etched PEO/PLLA blends, some lamellar branches inside the cracks underneath the bright band were twisted with some assuming an edge-on or flat-on arrangement. However, the external lamellar shells of the bright bands grew radially without turning, and they were only separated by oval-shaped cracks in the radial direction. Contrary to conventional conviction, twisting occurred inside the bright bands and not necessarily from the bright to the dark bands. In the dark band (valley), no cracks were observed. Figure 7 shows magnified AFM phase images for the ridge and valley regions of the water-etched PEO/PLLA (50/50) blend crystallized at $T_{\mathrm{c}}=110^{\circ} \mathrm{C}$, and the images are focused on the morphology of the lamellar regions away from the crack spots. Figures $7 \mathrm{~b}$ and $\mathrm{c}$ feature the ridge and valley regions, respectively. The AFM graph $(5 \mu \mathrm{m} \times 5 \mu \mathrm{m})$ of the ridge region $b$ shows a texture different from that of the valley region $c$. The surface patterns of the lamellae in the valley and ridge in these two regions appeared as flat-on and edge-on lamellae, respectively. The anisotropicity of the platelet crystals in these two regions was also apparently different. The orientation of the crystal lamellae in the valley (region c) was circumferential, whereas that of the lamellae in the ridge (region

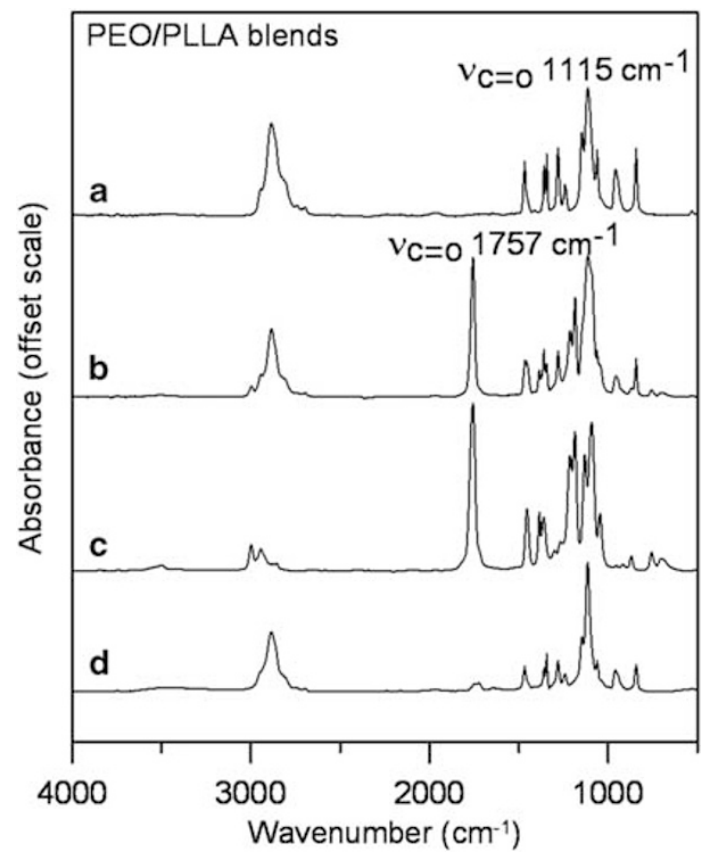

Figure 8 FTIR spectra for (a) neat PEO, (b) PEO/PLLA (50/50) blend, (c) neat PLLA and (d) the washed-out portion from PEO/PLLA (50/50) blend.

b) was radial. The difference in orientation in the ridge and valley regions may account for the cracks in the ridge regions but not in the valley region.

Finally it is essential to point out that washing on blend samples with water was effective in removing the PEO component from the crystallized PEO/PLLA blend and exposing the PLLA lamellae. To prove that the portion of the crystallized PLLA/PEO blend washed with water contained only PEO but PLLA remained mostly intact, FTIR observation was performed. Figures $8 \mathrm{a}-\mathrm{d}$ show the FTIR spectra for neat PEO, PEO/PLLA 50/50 blend, neat PLLA and the washed-out portion from the PEO/PLLA (50/50) blend, respectively. Spectrum 8 (d) clearly showed that the water-etched portion exhibited only the characteristic peaks of PEO and not of PLLA, indicating that only the PEO component was extracted. The very weak carbonyl peak at $1757 \mathrm{~cm}^{-1}$ in spectrum 8 (d) suggests that only a minor fraction of the amorphous PLLA was removed along with PEO during washing.

\section{CONCLUSIONS}

A critical insight into correlating cracks and ring-banded versus ringless spherulites in semicrystalline polymers has been obtained via the stepwise crystallization of two crystalline polymers: PEO and PLLA. The blends with high PEO contents crystallized into discrete and crystalline PEO spherulites that differed from PLLA spherulites that formed first during the crystallization process. However, the partial miscibility between PEO and PLLA caused some fractions of excess PEO to be trapped and later to crystallize in the interlamellae of the PLLA ring-banded spherulites. When the PEO was trapped in the PLLA ring bands, it tended to crystallize in (1) the concave portion of the PLLA lamellae and (2) the interlamellar crack regions of the bright bands (crystalline) band within the alternating ring bands of PLLA. Therefore, unetched PEO/PLLA blends exhibited flat surface topography with almost no vertical height variation on crystallization and 
cooling. They also had no interlamellar cracks in the convex lamellae (ridge) because the excess PEO filled the valley and the interlamellar crevices. The PLLA ring bands were intensified by the co-crystallization of PEO on PLLA because of the superimposed or partially overlapped crystals of PEO and PLLA.

The water-etched PEO/PLLA (50/50) blend exhibited periodical surface up and down topography with $3 \mu \mathrm{m}$ of vertical height variation. It displayed interlamellar cracks in the convex lamellae (ridge) because the excess PEO was etched off from the valley and the interlamellar crevices of the PLLA lamellae. The macro-lamellae outer plates $(\sim 20 \mu \mathrm{m}$ wide) exhibited up and down periodicity with flat-on orientation, but twisted micro-lamellae (1-2 $\mu \mathrm{m}$ wide) were visible inside the cracks on the ridge region. For the water-etched PEO/PLLA (80/20) blend, no interlamellar cracks and no macrolamellar up and down topography were present. AFM and SEM characterization revealed irregularly twisted thin micro-lamellae (1-2 $\mu \mathrm{m}$ wide) periodically interspersed with amorphous species, corresponding to POM graphs showing alternating and irregular zig-zag bands. Such micro-lamellae (1-2 $\mu \mathrm{m}$ wide) and their twisting patterns in the water-etched PEO/PLLA (80/20) blend resembled the sub-lamellae in the crack regions underneath the convex portion (ridge) of the up and down macro-lamellae in the water-etched PEO/ PLLA (50/50) blend. In addition, the AFM surface morphology of the water-etched blends revealed that the orientation of the crystal lamellae in the valley was circumferential, whereas that of lamellae in the ridge was radial. The difference in lamellae orientation in the ridge and valley regions could account for the alternating POM banding in the PEO/PLLA blend. It could also account for the interlamellar cracks in the ridge regions, but not the valley regions, of the PEO/PLLA (50/50) blend. Without the macro-lamellae in the PEO/PLLA (80/20) blend, no interlamellar cracks were present when the blend was cooled. However, inter-spherulitic cracks were still present.

\section{ACKNOWLEDGEMENTS}

This work has been financially supported by the basic research grants (NSC-972221-E-006-034-MY3) in consecutive years from the National Science Council of Taiwan, to which the authors express their gratitude.
1 Desai, N. P. \& Hubbell, J. A. Solution technique to incorporate polyethylene oxide and other water-soluble polymers into surfaces of polymeric biomaterials. Biomaterials 12, 144-153 (1991).

2 Desai, N. P. \& Hubbell, J. A. Surface physical interpenetrating networks of poly(ethylene terephthalate) and poly(ethylene oxide) with biomedical applications. Macromolecules 25, 226-232 (1992).

3 Lee, J. H., Kim, K. O. \& Ju, Y. M. Polyethylene oxide additive-entrapped polyvinyl chloride as a blood bag material. J. Biomed. Mater. Res. B 48, 328-334 (1999).

4 Chang, L. \& Woo, E. M. Effect of a miscible polymeric diluent on complex formation between isotactic and syndiotactic poly(methyl methacrylate). Ind. Eng. Chem. Res. 48, 3422-3440 (2009).

5 Pedrosa, P., Pomposo, J. A., Calahorra, E. \& Cortazar, M. On the glass transition behavior, interaction energies, and hydrogen-bonding strengths of binary poly(p-vinylphenol)/polyether blends. Macromolecules 27, 102-109 (1994).

6 Nakafuku, C. \& Sakoda, M. Melting and crystallization of poly(L-latic acid) and poly(ethylene oxide) binary mixture. Polym. J. 25, 909-917 (1993).

7 Nijenhuis, A. J., Colstee, E., Grijpma, D. W. \& Pennings, A. J. High molecular weight poly(L-lactic) and poly(ethylene oxide) blends: thermal characterization and physical properties. Polymer 37, 5849-5857 (1996).

8 Chee, M. J. K., Ismail, J., Kummerlöwe, C. \& Kammer, H. W. Study on miscibility of PEO and PCL in blends with PHB by solution viscometry. Polymer 43, 1235-1239 (2002).

9 Qiu, Z., Ikehara, T. \& Nishi, T. Unique morphology of poly(ethylene succinate)/ poly(ethylene oxide) blends. Macromolecules 35, 8251-8254 (2002).

10 Qiu, Z., Ikehara, T. \& Nishi, T. Miscibility and crystallization in crystalline/crystalline blends of poly(butylene succinate)/poly(ethylene oxide). Polymer 44, 2799-2806 (2003).

11 Agrawal, S. K., Sanabria-DeLong, N., Coburn, J. M., Tew, G. N. \& Bhatia, S. R. Novel drug release profiles from micellar solutions of PLA-PEO-PLA triblock copolymers. J. Control Release 112, 64-71 (2006).

12 Bergsma, J. E., Rozema, F. R., Bos, R. R. M., Boering, G., De Bruijn, W. C. \& Pennings, A. J. In vivo degradation and biocompatibility study of in vitro pre-degraded aspolymerized polylactide particles. Biomaterials 16, 267-274 (1995).

13 Barham, P. J. \& Keller, A. Relationship between microstructure and mode of fracture in polyhydroxybutyrate. J. Polym. Sci. A-2 Pol. Phys. 24, 69-77 (1986).

14 Martinez-Salazar, J., Sanchez-Cuesta, M., Barham, P. J. \& Keller, A. Thermal expansion and spherulite cracking in 3-hydroxybutyrate/3-hydroxyvalerate copolymers. J. Mater. Sci. Lett. 8, 490-492 (1989).

15 Hobbs, J. K., McMaster, T. J., Miles, M. J. \& Barham, P. J. Cracking in spherulites of poly(hydroxybutyrate). Polymer 37, 3241-3246 (1996).

16 Xu, J., Guo, B. H., Chen, G. Q. \& Zhang, Z. M. Terraces on banded spherulites of polyhydroxyalkanoates. J. Polym. Sci. Pol. Phys. 41, 2128-2134 (2003).

17 Kuboyama, K. \& Ougizawa, T. Special issues-International symposium on polymer crystallization 2007-Solvent induced cracking and morphology in banded spherulite of poly(trimethylene terephthalate). Polym. J. 40, 1005-1009 (2008).

18 Nakafuku, C. Effects of molecular weight on the melting and crystallization of poly (L-lactic acid) in the mixture with poly(ethylene oxide). Polym. J. 28, 568-575 (1996).

19 Nurkhamidah, S. \& Woo, E. M. Effect of crystallinity and molecular weight on crack behavior in crystalline poly(L-lactic acid). J. Appl. Polym. Sci. (doi:10.1002/app.34021).

20 Nurkhamidah, S. \& Woo, E. M. Cracks and ring bands of poly(3-hydroxybutyrate) on precrystallized poly(L-lactic acid) template. Ind. Eng. Chem. Res. 50, 4494-4503 (2011). 